\title{
The Rapid Effect of Bisphenol-A on Long-Term Potentiation in Hippocampus Involves Estrogen Receptors and ERK Activation
}

\author{
Xiaowei Chen, ${ }^{1}$ Yu Wang, ${ }^{1,2}$ Fang Xu, ${ }^{1}$ Xiaofei Wei, ${ }^{1}$ Junfang Zhang, ${ }^{1}$ \\ Chuang Wang, ${ }^{1}$ Hua Wei, ${ }^{1}$ Shujun Xu, ${ }^{1}$ Peiyun Yan, ${ }^{1}$ Wenhua Zhou, ${ }^{1}$ Istvan Mody, \\ Xiaohong $\mathrm{Xu}^{2}$ and Qinwen Wang ${ }^{1}$ \\ ${ }^{1}$ Ningbo Key Laboratory of Behavioral Neuroscience, Zhejiang Provincial Key Laboratory of Pathophysiology, School of Medicine, \\ Ningbo University, Ningbo 315211, China \\ ${ }^{2}$ Chemistry and Life Sciences College, Xingzhi College, Zhejiang Provincial Key Laboratory of Ecology, Zhejiang Normal University, \\ Jinhua 321004, China
}

Correspondence should be addressed to Xiaohong Xu; xuxh63@zjnu.cn and Qinwen Wang; wangqinwen@nbu.edu.cn

Received 12 September 2016; Revised 11 December 2016; Accepted 25 December 2016; Published 31 January 2017

Academic Editor: Luis J. Santín

Copyright (C) 2017 Xiaowei Chen et al. This is an open access article distributed under the Creative Commons Attribution License, which permits unrestricted use, distribution, and reproduction in any medium, provided the original work is properly cited.

\begin{abstract}
Bisphenol-A (BPA), a widely used synthetic compound in plastics, disrupts endocrine function and interferes with physiological actions of endogenous gonadal hormones. Chronic effects of BPA on reproductive function, learning and memory, brain structure, and social behavior have been intensively investigated. However, less is known about the influence of BPA on long-term potentiation (LTP), one of the major cellular mechanisms that underlie learning and memory. In the present study, for the first time we investigated the effect of different doses of BPA on hippocampal LTP in rat brain slices. We found a biphasic effect of BPA on LTP in the dentate gyrus: exposure to BPA at a low dose $(100 \mathrm{nM})$ enhanced LTP and exposure to BPA at a high dose $(1000 \mathrm{nM})$ inhibited LTP compared with vehicle controls. The rapid facilitatory effect of low-dose BPA on hippocampal LTP required membrane-associated estrogen receptor (ER) and involved activation of the extracellular signal-regulated kinase (ERK) signaling pathway. Coadministration of $17 \beta$-estradiol $\left(\mathrm{E}_{2}\right.$, the primary estrogen hormone) and BPA (100 nM) abolished both the BPA-induced enhancement of LTP and the $\mathrm{E}_{2}$-induced enhancement of baseline fEPSP, suggesting a complex interaction between BPA- and $\mathrm{E}_{2}$-mediated signaling pathways. Our investigation implies that even nanomolar levels of endocrine disrupters (e.g., BPA) can induce significant effects on hippocampal LTP.
\end{abstract}

\section{Introduction}

Bisphenol-A (BPA) is a widely used synthetic compound included in polycarbonate plastics and epoxy resins, for example, in food and beverage containers, dental prostheses, compact discs, and baby bottles. It is capable of acting as an endocrine disrupter and interferes with actions of endogenous gonadal hormones (e.g., estrogen or androgen) at low concentrations. BPA can bind to estrogen receptors (ERs) at low concentration and thus affects normal hormonal regulation and endocrine function [1]. A large number of studies have indicated that chronic exposure to the lowdose (nanomolar) BPA during fetal/neonatal stages inhibits sexual differentiation and nonreproductive behaviors of adult animals [2-4].
Although the widespread effects of BPA on reproductive function, brain structure, and social behavior have been investigated, recent studies reported controversial actions of BPA on learning and memory, ranging from deficits to no effect and to enhancements. In rodents, pre- and perinatal exposures to BPA at or below the TDI (tolerable daily intake; $\leqslant 50 \mu \mathrm{g} / \mathrm{kg} /$ day) have resulted in adverse effects on memory processes [4-9]. Adolescent exposure to BPA below the TDI impairs spatial memory in rats [5]. In contrast, other studies have shown that chronic oral exposure to BPA does not alter memory processes of adult male or ovariectomized (OVX) female rats $[10,11]$. We previously found that acute exposure to BPA rapidly enhanced short-term passive avoidance memory in the developing rats [12]. The underlying mechanism is unclear. The role of BPA in synaptic remodeling in brain 
areas involved in learning and memory is also controversial. Adolescent exposure to low-dose BPA inhibited spinogenesis and synaptic modification in hippocampi of rodents [13]. BPA inhibited $17 \beta$-estradiol $\left(\mathrm{E}_{2}\right)$-induced formation of dendritic spine synapses in hippocampal CA1 area and prefrontal cortex of adult ovariectomized rats or nonhuman primates $[14,15]$. However, other studies have shown the facilitatory effects of BPA on synaptic plasticity in neuronal development. Exposure to BPA at low doses $(<100 \mathrm{nM})$ enhanced both dendritic and synaptic development in cultured hypothalamic cells $[16,17]$. Exposure to BPA at $10-100 \mathrm{nM}$ for 30 min rapidly increased the spine density dendritic filopodia mobility of the hippocampus [18]. Nanomolar doses of BPA rapidly modulated spinogenesis in adult hippocampal neurons [19]. Our previous study has also identified the facilitatory effect of BPA on dendritic morphogenesis of cultured hippocampal neurons through ER activation [12].

The long-lasting plasticity of synaptic transmission, as long-term potentiation (LTP) or long-term depression (LTD), is thought to be the cellular basis of learning and memory processes. Interestingly, it has been reported that exposure to BPA at low concentrations (10-100 nM) rapidly enhanced LTD in CA1 and CA3 but suppressed LTD in the dentate gyrus of the hippocampus [20, 21]. However, no studies have assessed the potential of BPA to influence LTP, and the underlying mechanisms are yet largely unknown.

The extracellular signal-regulated kinase (ERK) signal pathway is a component of a mitogen-activated protein kinase (MAPK) signaling cascade which regulates a variety of important cellular events. Recently, evidence highlights the ERK-mediated effects of estrogen and xenoestrogens in the brain [22]. Our previous studies have demonstrated that ERK signaling is involved not only in the chronic effect of BPA on dendritic morphogenesis in hippocampal neurons but also in the rapid effect of BPA on passive avoidance memory of young rats $[12,23]$.

In the present study, we investigated the dose-dependent effect of BPA on hippocampal LTP and explored the downstream intracellular pathways. In addition, we examined the synergistic role of BPA and $\mathrm{E}_{2}$ in hippocampal LTP. Therefore our study provides additional information on possible mechanisms for the effects of BPA on synaptic plasticity in brains.

\section{Materials and Methods}

2.1. Animal and Drug Treatment. All experiments were carried out on male Wistar rats (Weight $120-140$ g, age 5-6 weeks). The use of animals for experimental procedures was carried out in accordance with Guidelines for the Care and Use of the Laboratory Animals of Ningbo University, China.

2.2. Preparation of Slices. All experiments were conducted on transverse slices of the rat hippocampus. The brains were rapidly removed after decapitation and placed in cold oxygenated $\left(95 \% \mathrm{O}_{2}, 5 \% \mathrm{CO}_{2}\right)$ artificial cerebral spinal fluid (ACSF). Slices were cut at a sickness of $350 \mu \mathrm{m}$ using a VT $1000 \mathrm{~S}$ vibroslicer (Leica, Germany) and placed in a storage chamber containing oxygenated medium at room temperature $\left(20-22^{\circ} \mathrm{C}\right)$ for $1 \mathrm{~h}$. The slices were then transferred to a recording chamber and continuously superfused at a rate of $5-6 \mathrm{~mL} / \mathrm{min}$ at $30-32^{\circ} \mathrm{C}$. The ACSF contained (mM) $\mathrm{NaCl}, 120 ; \mathrm{KCl} 2.5, \mathrm{NaH}_{2} \mathrm{PO}_{4}, 1.25 ; \mathrm{NaHCO}_{3} 26 ; \mathrm{MgSO}_{4}$, $2.0 ; \mathrm{CaCl}_{2}, 2.0$; D-glucose 10 . All solutions contained $100 \mu \mathrm{M}$ picrotoxin (Sigma, St Louis, MO, USA) to block GABA $_{\mathrm{a}}$ mediated activity.

2.3. In Vitro Electrophysiological Techniques. The electrophysiological techniques were applied according to our previous reports $[24,25]$. Presynaptic stimulation was applied to the medial perforant pathway of the dentate gyrus using a bipolar insulated tungsten wire electrode, and field excitatory postsynaptic potentials (fEPSPs) were recorded at a control test frequency of $0.033 \mathrm{~Hz}$ from the middle one-third of the molecular layer of the dentate gyrus with a glass microelectrode. The inner blade of the dentate gyrus was used in all studies. In each experiment, an input-output curve (afferent stimulus intensity versus fEPSP amplitude) was plotted at the test frequency. For all experiments, the amplitude of the test EPSP was adjusted to one-third of maximum $(\sim 1.2 \mathrm{mV})$. LTP was evoked by high-frequency stimulation (HFS) consisting of two trains (each of two stimuli at $100 \mathrm{~Hz}$ for $1 \mathrm{~s}$, intertrain interval $15 \mathrm{~s}$ ) with the stimulation voltage increased during the HFS so as to evoke an initial EPSP of the train of double the normal test EPSP amplitude.

2.4. Statistics. Recordings were analyzed using pCLAMP 10.3 software (Axon Instruments, Foster City, CA, USA). Values are the means \pm SEM for $n$ slices. All brain slices in the same group were from different animals. In most experiments, the amplitude of fEPSPs measured $40 \mathrm{~min}$ after HFS (postHFS) was shown, unless indicated otherwise. Two-tailed Student's $t$-test and one-way ANOVA were used for the detailed statistical analysis where appropriate; $p<0.05$ was considered statistically significant.

2.5. Agents. All drugs were applied through the perfusion medium. BPA was purchased from Shanghai Chemical Reagent Research Institute (Shanghai, China). $17 \beta-\mathrm{E}_{2}$ and U0126 were purchased from Cell Signaling (Boston, MA, USA). ICI182,780 was purchased from Tocris (Ballwin, MO, USA). All reagents were dissolved in dimethyl sulphoxide (DMSO, from Sigma, St. Louis, MO, USA) and then diluted in ACSF (0.05\% vehicle). Control levels of LTP were measured on slices perfused with vehicle (DMSO) alone.

\section{Results}

3.1. The Facilitatory Effect of Low-Dose BPA on LTP in the Dentate Gyrus. We first investigated the dose-dependent effect of BPA $(10,100$, and $1000 \mathrm{nM}$; added to the ASCF 60 min before HFS) on synaptic plasticity of perforant pathgranule cell synapses induced by HFS in the dentate gyrus (DG). We found that application of $10 \mathrm{nM} \mathrm{BPA}$ did not have any effect on LTP $(140.8 \pm 5.2 \%$ of baseline, $n=8)$ compared with vehicle controls $(143.7 \pm 7.6 \%$ of baseline, $n=8, p>$ 0.05 , Figures $1(\mathrm{a})$ and $1(\mathrm{~b}))$. However, $100 \mathrm{nM}$ BPA increased LTP $(193.1 \pm 8.3 \%$ of baseline, $n=8)$ compared to control 

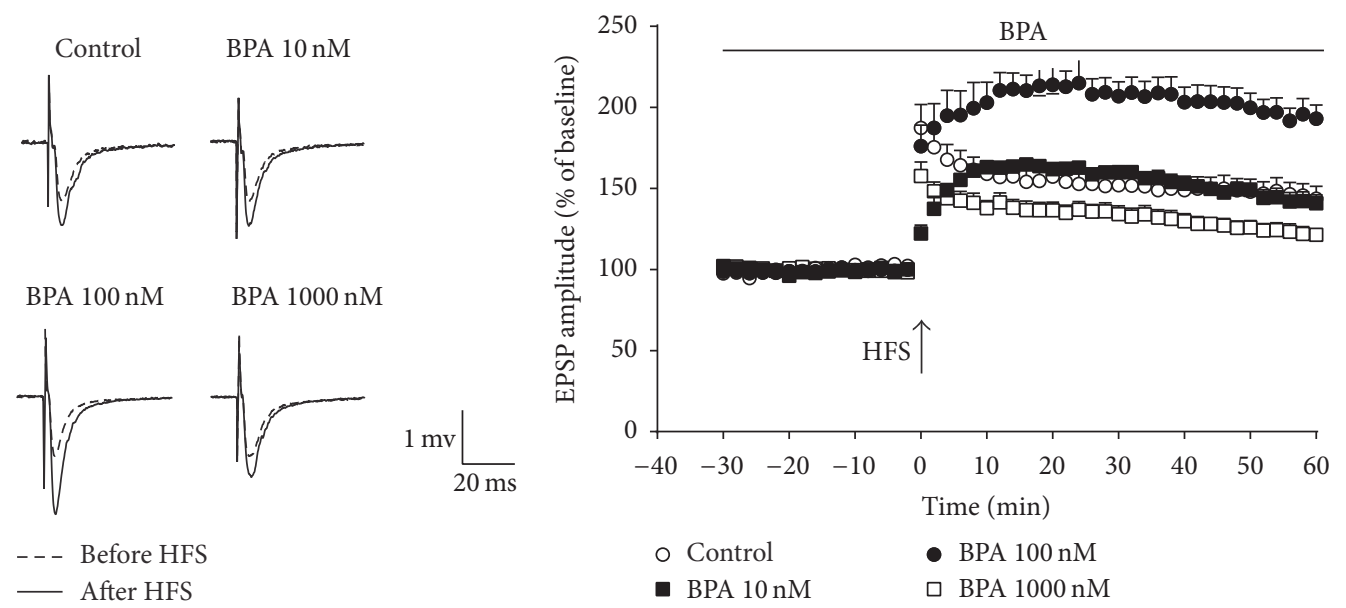

(a)

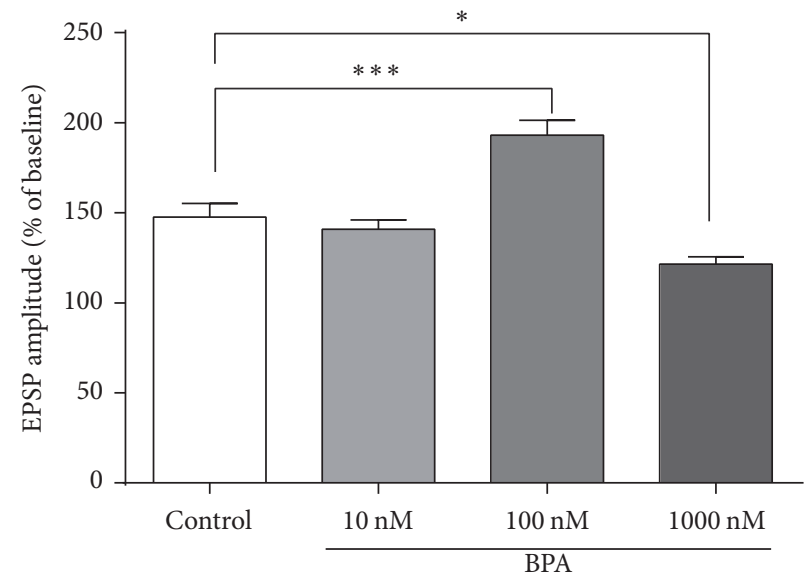

(b)

FIGURE 1: The biphasic effect of BPA on LTP in rat dentate gyrus in vitro. (a) High-frequency stimulation induced LTP in the medial perforant path of the dentate gyrus of acute rat hippocampus slices (open circles, $n=8$ ). Applications of BPA are indicated at concentrations of $10 \mathrm{nM}$ (filled squares, $n=8$ ), $100 \mathrm{nM}$ (filled circles, $n=8$ ), and $1000 \mathrm{nM}$ (open squares, $n=8$ ), respectively. All hippocampal slices were preperfused with ACSF, $30 \mathrm{~min}$ before HFS, to obtain baseline EPSP amplitude. (b) Summary of the major experimental outcomes. The average fEPSP amplitudes at $60 \mathrm{~min}$ after HFS in separate perfusion of different concentration BPA. Applications of BPA $100 \mathrm{nM}$ and BPA $1000 \mathrm{nM}$ have significant effects on LTP, ${ }^{*} p<0.05,{ }^{* * *} p<0.001$ as compared to controls. Solid and dashed example traces before HFS and after HFS, respectively.

$(143.7 \pm 7.6 \%$ of baseline, $n=8, p<0.001$, Figures 1 (a) and 1(b)). In contrast, application of BPA $1000 \mathrm{nM}$ resulted in an inhibition of LTP in DG $(121.1 \pm 4.0 \%$ of baseline, $n=8$, $p<0.05$, Figure 1(b)), indicating a biphasic effect of low-dose (100 nM) and high-dose (1000 nM) BPA on hippocampal LTP.

3.2. The BPA-Enhanced LTP Requires Activation of ERs. To examine whether the enhancement of LTP by $100 \mathrm{nMBPA}$ involves ERs, we add a high-affinity nonselective ER antagonist ICI 182,780 (100 nM) into bath solution $30 \mathrm{~min}$ before BPA application. Application of ICI 182,780 had no effect on LTP (120.6 $\pm 3.7 \%$ of baseline, $n=8$, controls: $140.8 \pm 5.2 \%$ of baseline, $n=8 . p>0.05$, Figure 2(b)) but blocked BPAenhanced LTP $(123.4 \pm 6.2 \%$ of baseline, $n=8, p<0.001$, Figure 2(b)), suggesting that the facilitatory effect of BPA $(100 \mathrm{nM})$ on LTP in hippocampal dentate gyrus requires the activation of ERs.
3.3. BPA-Enhanced LTP Involves ERKs. To explore the downstream signaling pathway of the BPA-enhanced LTP in rat hippocampus, we examined whether the ERK pathway is involved. Application of 100 nM U0126 (a MEK1/2 or ERK inhibitor) $60 \mathrm{~min}$ before HFS did not alter the baseline fEPSP but inhibited the hippocampus LTP in rat dentate gyrus compared with vehicle controls (103.1 $\pm 3.5 \%$ of baseline, $n=8, p<0.001$, Figures 3(a) and 3(c)). In addition, pretreatment of $100 \mathrm{nM}$ U0126 added $30 \mathrm{~min}$ before BPA application completely blocked BPA-enhanced LTP $(102.8 \pm 6.1 \%$ of baseline, $n=8, p<0.001$, Figure $3(\mathrm{c}))$. However, pretreatment of BPA (added $30 \mathrm{~min}$ before U0126 application) resulted in partial inhibition of BPA-enhanced LTP $(151.0 \pm 4.7 \%$ of baseline, $n=8, p<0.001$, Figure $3(\mathrm{~d}))$. These results indicate that activation of ERK pathway is not only required for physiological LTP but also necessary for the facilitatory effect of BPA on LTP in the dentate gyrus. 

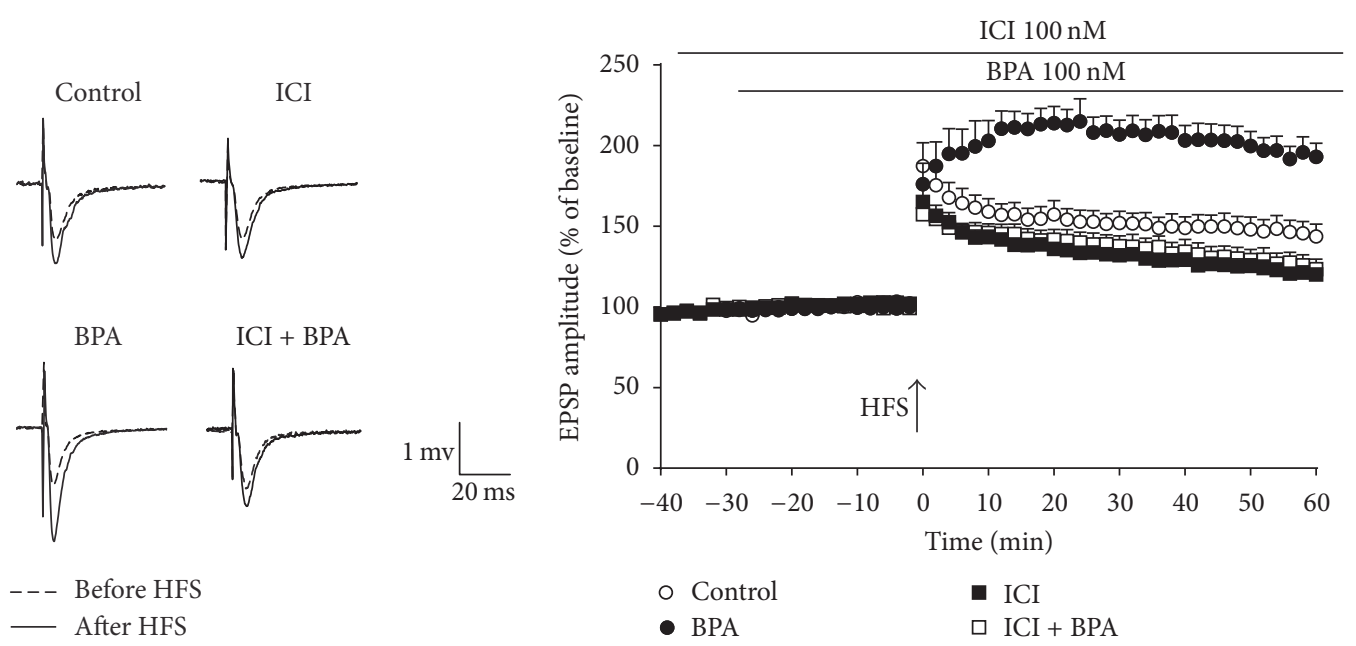

(a)

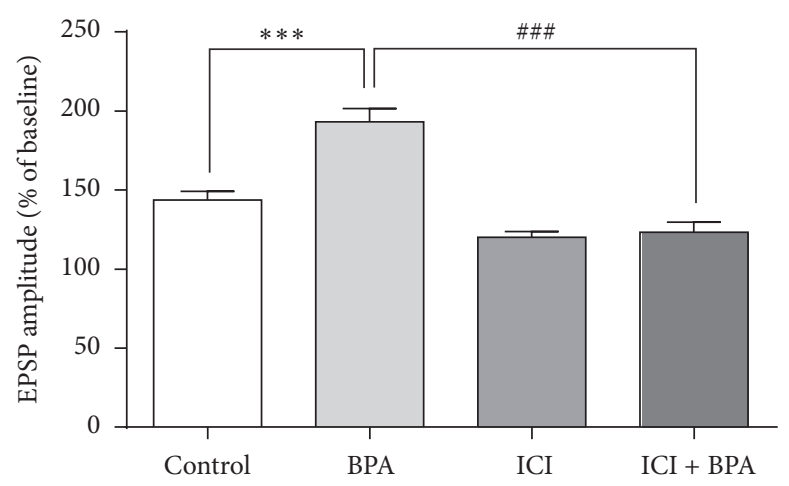

(b)

Figure 2: The enhancement of BPA on hippocampal LTP was ER-dependent. (a) Administration of ICI 182,780 $10 \mathrm{nM}$ (an antagonist of ERs, filled square, $n=8$ ) remarkably decreased the $100 \mathrm{nM} \mathrm{BPA}$-induced enhancement of LTP. Pretreatment with the ERs antagonist ICI $182,78030 \mathrm{~min}$ before BPA $100 \mathrm{nM}$ (open squares, $n=8$ ) application completely blocked BPA-enhanced LTP compared with BPA alone. (b) Figure columns express the average fEPSP amplitudes after HFS in separate perfusion or coperfusion of BPA $100 \mathrm{nM}$ and ICI $182,780100 \mathrm{nM}$, ${ }^{* * *} p<0.001$ as compared to the control, ${ }^{\# \#} p<0.001$ as compared to the BPA $100 \mathrm{nM}$. Solid and dashed example traces before HFS and after HFS, respectively.

3.4. The Effects of BPA and $E_{2}$ on Baseline fEPSP and LTP Enhancement. Previous studies have reported the facilitatory effect of $\mathrm{E}_{2}$ on both baseline fEPSP and LTP induction [26]. Here, we applied $10 \mathrm{nM} \mathrm{E}_{2}$ on rat brain slice and observed a significant increase ( $20-30 \%)$ of baseline fEPSP compared with vehicle controls (Figures 4(a) and 4(b)). However, coapplication of BPA (100 nM) and $\mathrm{E}_{2}$ reversed the enhancement of baseline fEPSP induced by $\mathrm{E}_{2}$ (Figure $4(\mathrm{~b})$ ). In terms of LTP enhancement, $\mathrm{E}_{2}$ treatment did not enhance LTP while comparing fEPSP before HFS (at $0 \mathrm{~min}$ ) and after HFS (at $60 \mathrm{~min}$ ) in the dentate gyrus (Figures 4(b) and 4(c)). Unexceptionally, coapplication of $\mathrm{E}_{2}$ and BPA blocked both the BPA-induced enhancement of LTP and the $\mathrm{E}_{2}$-induced enhancement of baseline fEPSP (Figures 4(b) and 4(c)).

\section{Discussion}

4.1. The Rapid Facilitatory Effect of Low-Dose BPA on Hippocampal LTP Is ER-Dependent and Involves Activation of ERK Pathway. The rapid effect of BPA on synaptic plasticity has been investigated by several studies. It is shown that lowdose BPA (10 nM) increases Ca influx, enhances filopodia flexibility in cultured hippocampal neurons, and rapidly modulates spinogenesis in adult hippocampal slices [18, 19]. These effects have been reported to relate to ERs and MAPK activation [19]. In the terms of memory-related synaptic plasticity (e.g., LTP and LTD), the effect of BPA has been less investigated. Hasegawa et al. [21] have reported the BPA-induced enhancement of LTD in CAl region of rat hippocampus, but this effect does not require ER activation [21]. However, we here demonstrate that low-dose BPA $(100 \mathrm{nM})$ significantly enhances LTP in rat DG region and this facilitatory effect of BPA on LTP depends on ER activation since $\mathrm{E}_{2}$ antagonist ICI 182,780 completely abolishes the BPA enhancement on LTP.

There are two types of ERs: one type is nuclear estrogen receptors (nERs), which are members of the nuclear receptor family of intracellular receptors, including ER $\alpha$ and ER; the other type is membrane estrogen receptors (mERs), which are mostly $\mathrm{G}$ protein-coupled receptors, including Gq-coupled 

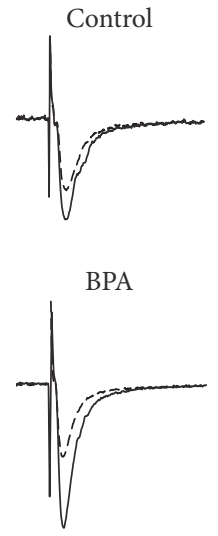

- - - Before HFS

— After HFS
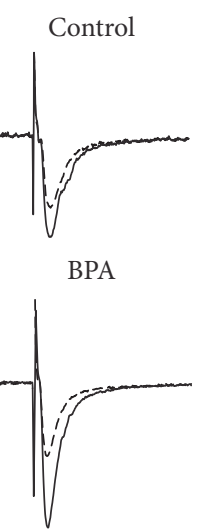

-- - Before HFS

- After HFS

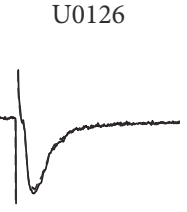

$\mathrm{U} 0126+\mathrm{BPA}$

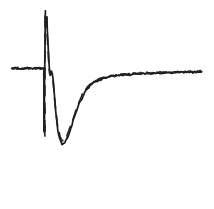

U0126

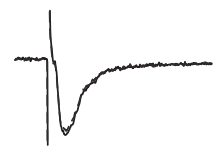

$\mathrm{BPA}+\mathrm{U} 0126$

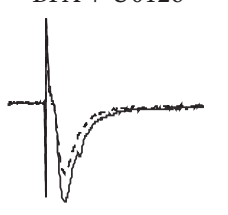

$1 \mathrm{mv}$

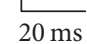

$20 \mathrm{~ms}$

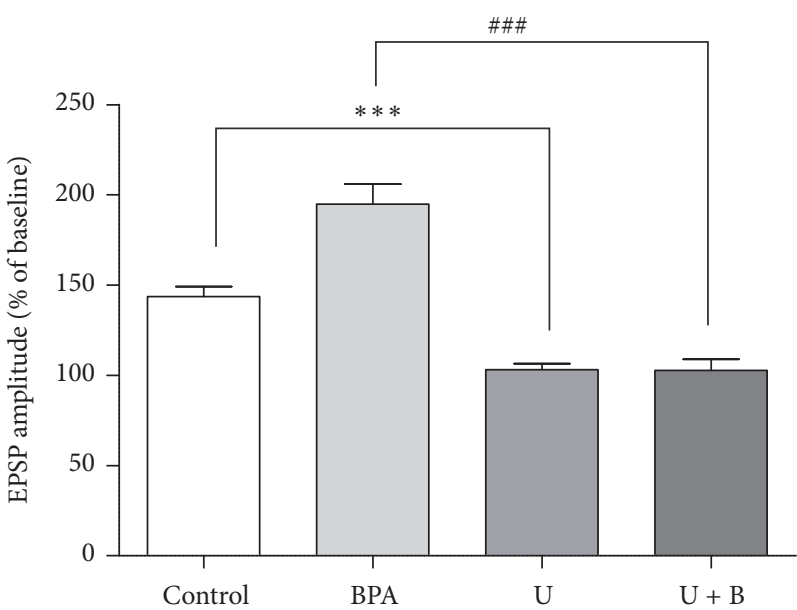

(c)

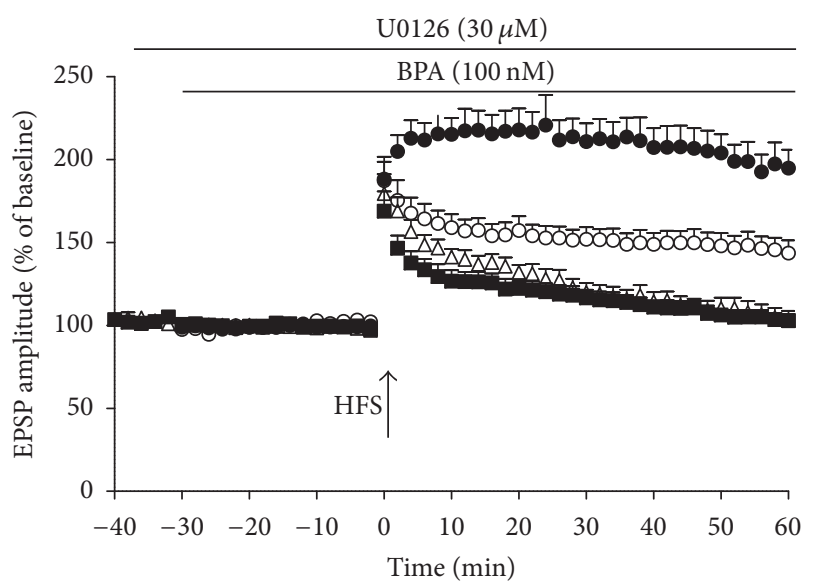

O Control

- U0126

- BPA

$\triangle \mathrm{U} 0126+\mathrm{BPA}$

(a)

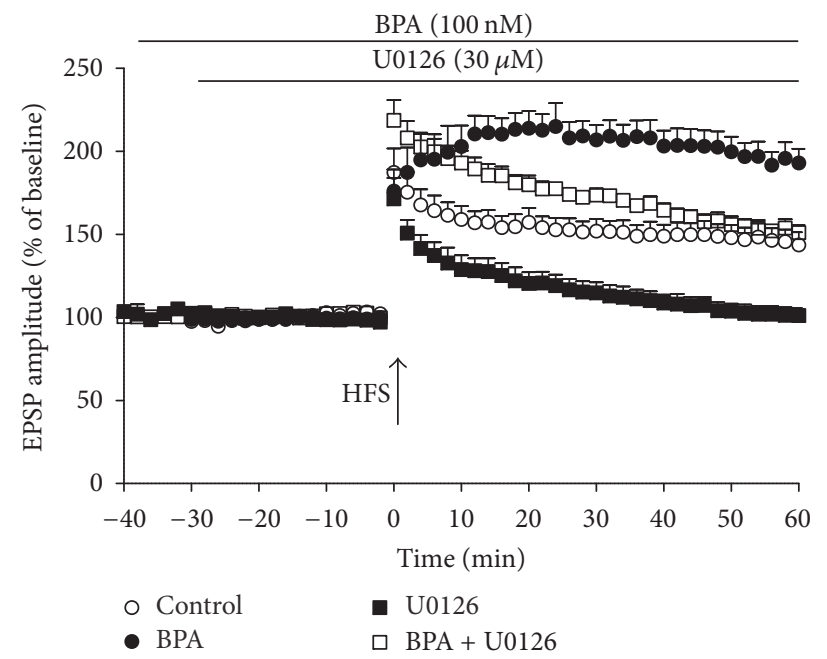

(b)

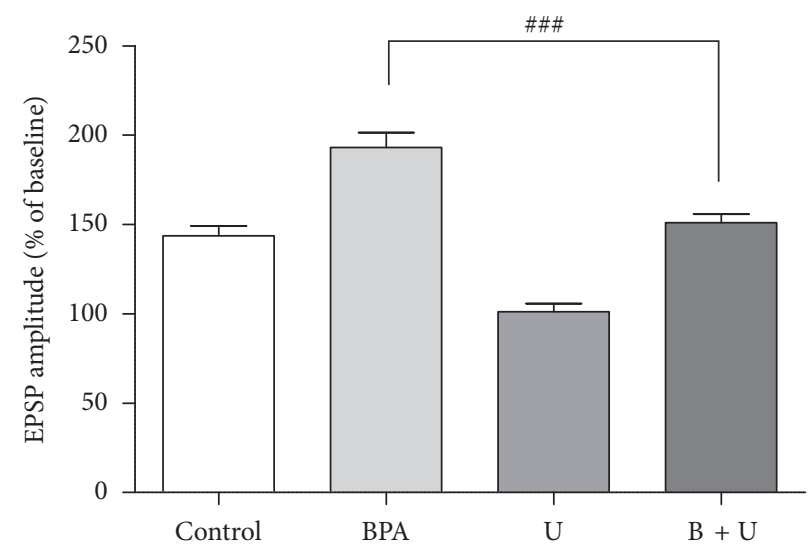

(d)

FIGURE 3: ERK signal pathway was involved in BPA-enhanced LTP. Pretreatment with ERK inhibitor U0126 for 30 min before BPA $100 \mathrm{nM}$ (open triangles, $n=8$ ) completely blocked LTP compared with controls. (b) Pretreatment with BPA $100 \mathrm{nM} 30$ min before the ERK inhibitor (open squares, $n=8$ ) application remarkably decreased the BPA effect as compared with BPA $100 \mathrm{nM}$ alone (open squares). (c, d) Figure columns showing the average fEPSP amplitudes at $60 \mathrm{~min}$ after HFS in separate perfusion or coperfusion of BPA $100 \mathrm{nM}$ and U0 $12630 \mu \mathrm{M}$, ${ }^{* * *} p<0.001$ as compared to the control, ${ }^{\# \# \#} p<0.001$ as compared to the BPA $100 \mathrm{nM}$. Solid and dashed example traces before HFS and after HFS, respectively. 


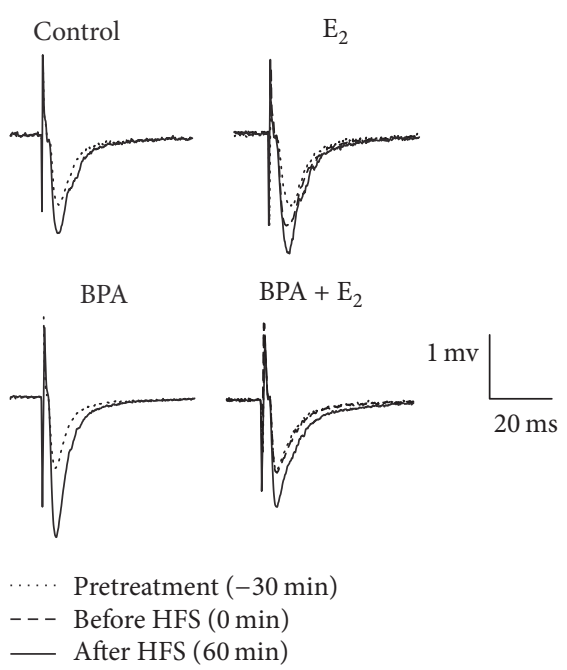

Pre-HFS

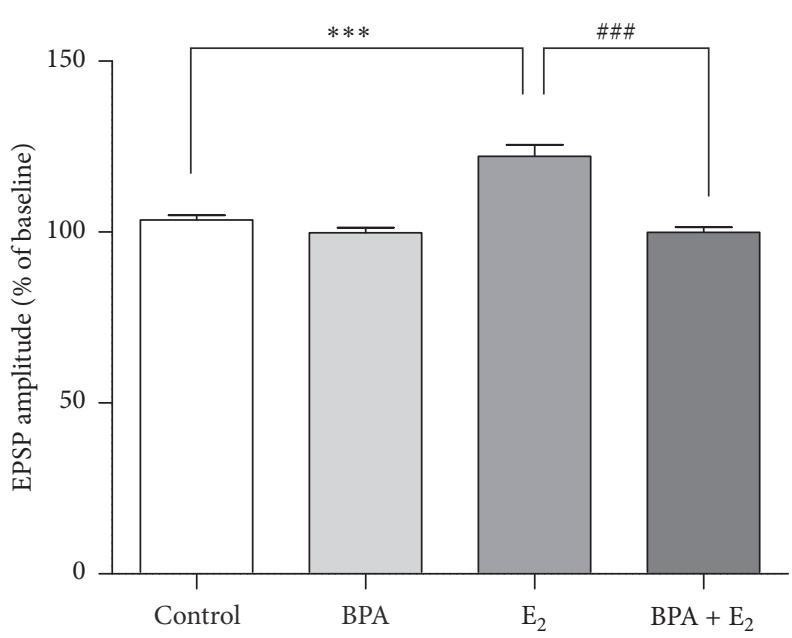

(b)

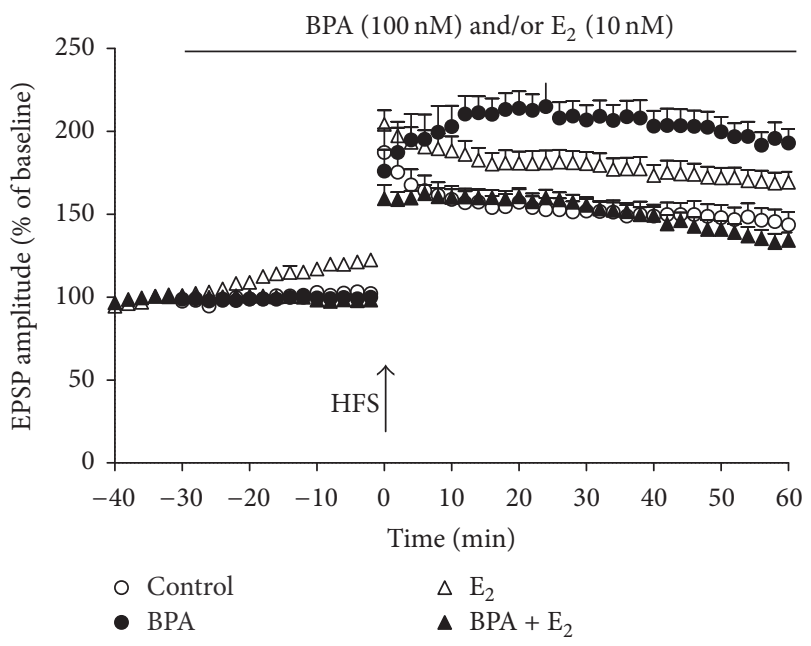

(a)

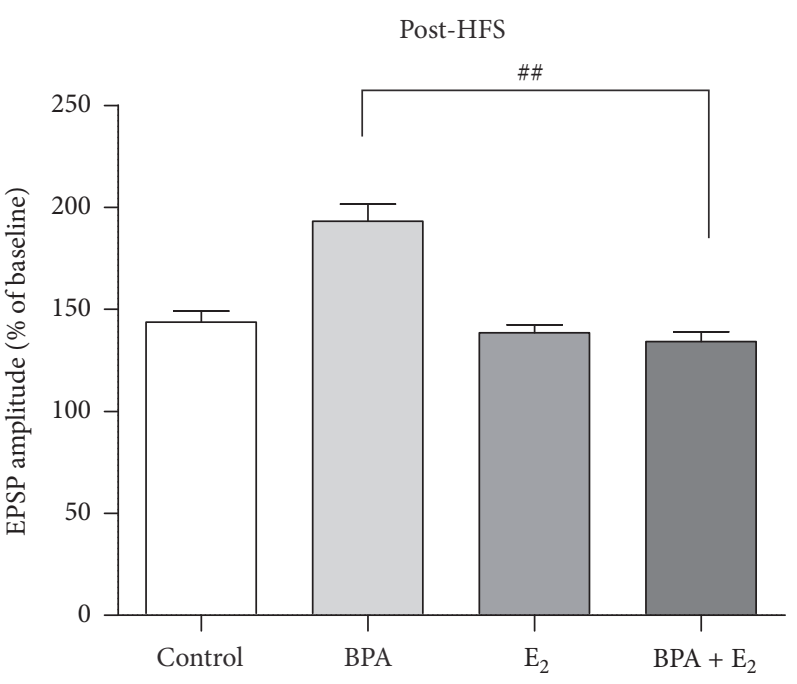

(c)

FIGURE 4: The enhancement of BPA on hippocampal LTP was abolished by $\mathrm{E}_{2}$ treatment. Enhanced LTP by $\mathrm{E}_{2}(10 \mathrm{nM})$ in hippocampal area $\mathrm{DG}$ (open triangles, $n=8$ ). Coadministration of BPA $100 \mathrm{nM}$ with $\mathrm{E}_{2}$ (filled triangles, $n=8$ ) had no effect on LTP compared with untreated controls. (b) Pretreatment by $\mathrm{E}_{2}$ enhanced the pre-HFS EPSP amplitudes, ${ }^{* * *} p<0.001$ as compared to controls, ${ }^{\# \# *} p<0.001$ as compared to the $\mathrm{E}_{2} 10 \mathrm{nM}$. (c) Comparison of different groups the fEPSP amplitudes at $60 \mathrm{~min}$. $\mathrm{E}_{2}$ had no effect on LTP compared with untreated controls without baseline increase in EPSP amplitude (before the HFS). ${ }^{* * *} p<0.001$ as compared to the control, ${ }^{\# *} p<0.01$ as compared to the BPA $100 \mathrm{nM}$. Solid and dashed traces are examples of before treatment, after treatment, and after HFS, respectively.

mER (Gq-ER), GPER1 (formerly GPR30), and ER-X [27]. In the genomic mechanism, $\mathrm{E}_{2}$ binds to $\mathrm{ER} \alpha$ and $\mathrm{ER} \beta$ in the cytoplasm, and then the $\mathrm{E}_{2}$-ER complex translocates into the nucleus, binds to an estrogen response element on the DNA, and finally facilitates gene transcription. The nongenomic mechanism involves actions of mERs at the plasma membrane: ER $\alpha$ and ER $\beta$ interact with mERs to rapidly activate extracellular signal-regulated kinase (ERK) cell signaling, which further triggers epigenetic processes, gene expression, and other cell signaling pathways [22]. Although it is not clear which type of ER(s) is involved in the facilitatory effect of BPA on LTP because ICI 182,780 blocks both nERs and mERs, the rapid effect of BPA (within $1 \mathrm{~h}$ ) indicates a greater contribution of the nongenomic mER signaling to the BPA-induced enhancement of hippocampus LTP. Considering the essential roles of glutamate receptors (AMPA, NMDA, and metabotropic glutamate receptor) in the hippocampal LTP and the interactions between glutamate receptors and mERs, the glutamate receptors may also be involved in BPA-induced enhancement of LTP.

Growing evidence demonstrates that the hippocampal ERK signaling is necessary for $\mathrm{E}_{2}$ to enhance hippocampal memory consolidation [28, 29]. Here our results confirm that ERK activation is also required for the BPA-induced enhancement of LTP. It is interesting that the blockade of ERK pathway did not completely inhibit BPA-enhanced LTP while the slices were preincubated with BPA for 30 min before U0126 treatment. The reason may be due to the rapid effect of 
BPA on LTP since preincubation of BPA may already launch certain rapid downstream effects to enhance EPSP amplitude after high-frequency stimulation, whereas some slow effects of BPA requiring ERK activation are inhibited by following the application of U0126. These results are consistent with our previous findings on cultured rat hippocampal neurons that exposure to BPA for $30 \mathrm{~min}$ rapidly enhances the motility and the density of dendritic filopodia through the ER-mediated pathway [30]. Finally, our results also show that highdose BPA (1000 nM) could severely inhibit hippocampal LTP, indicating a complex mechanism of BPA actions on neuroplasticity in hippocampi.

4.2. BPA and $E_{2}$ Differently Influence Hippocampal LTP and There Might Be a Complex Interaction between Them. Estrogen (e.g., $\mathrm{E}_{2}$ ) is also locally synthesized within the hippocampus in addition to the gonads. Mounting articles demonstrate that $\mathrm{E}_{2}$ influences hippocampal memory [31, 32]. A number of studies have reported rapid effects of $E_{2}$ on LTP, LTD, and spinogenesis in the hippocampus. Low concertation of $\mathrm{E}_{2}(1 \mathrm{nM})$ rapidly enhances LTD in CA1, CA3, and dentate gyrus of the hippocampus. The density of thin type spines increases in CA1 pyramidal neurons within $2 \mathrm{~h}$ after application of $1 \mathrm{~nm}$ estradiol and this enhancement of spinogenesis requires ERs and MAPK signals [33]. Vedder et al. have demonstrated that $\mathrm{E}_{2}$-induced enhancements in both spatial memory and LTP occur within a similar time frame, linking $\mathrm{E}_{2}$-induced changes in LTP with hippocampal memory formation [34]. Our previous study also confirms that $E_{2}(10 \mathrm{nM})$ significantly increases the total dendritic length and enhances motility and density of dendritic filopodia in cultured hippocampal neurons [12]. In the terms of hippocampal LTP, although application of $\mathrm{E}_{2}(1-10 \mathrm{nM})$ does not directly enhance LTP, it induces a baseline increase of the excitatory postsynaptic potential (EPSP) in CA1 neurons $[26,35]$.

Consistently, in the present study, we have shown a significant increase ( 20-30\%) of baseline fEPSP induced by application of $\mathrm{E}_{2}$. Molecular mechanisms of modulation through synaptic estrogen receptor (ER) and its downstream signaling are still unknown. It may involve a complex kinase network based on a recent study investigating the induction of LTP by the presence of $\mathrm{E}_{2}$ upon weak theta burst stimulation (a subthreshold stimulation that did not induce full-LTP) in CA1 region of the adult male hippocampus [36]. This $\mathrm{E}_{2}$-induced LTP is ER-dependent and requires activation of multiple kinases including ERK, protein kinase A (PKA), protein kinase C (PKC), phosphatidylinositol 3kinase (PI3K), and calcium calmodulin kinase II (CaMKII) [36].

It is worth noting that although exposure to either low-dose BPA or $\mathrm{E}_{2}$ alone enhances LTP suppression of these effects is observed when low-dose BPA and low-dose $\mathrm{E}_{2}$ are administrated together. Our findings are consistent with a previous in vivo study showing that the $\mathrm{E}_{2}$-induced increase in synapse density is inhibited by the simultaneous application of BPA $(40 \mathrm{ug} / \mathrm{kg})$ and $\mathrm{E}_{2}(60 \mathrm{ug} / \mathrm{kg})$ in ovariectomized rats for $30 \mathrm{~min}$ [37]. The underlying mechanism is still unclear and requires further exploration. A possible explanation might be the influence of the allosteric effect of BPA on ERs. Binding of BPA to ERs may change the structure of $E_{2}$ binding sites and affect the affinity of $E_{2}$ to ERs. However, recent studies highlight another possibility that fluctuations of local $\mathrm{E}_{2}$ levels during a learning event may be a key factor in learning and memory [32]. A study in adult nonhuman primates reported that elevated $\mathrm{E}_{2}$ level by applying exogenous $\mathrm{E}_{2}$ interferes with a cognitive function on the delayed response task in female monkeys [38]. Nevertheless, a study in finches has found that dynamic suppression of $E_{2}$ synthesis during a learning event may be a critical component of learning processes [39]. Possibly, low-dose BPA alone may act as the ER modulator and has estrogenlike effects on synaptic plasticity in the hippocampus, whereas high-dose BPA alone may act as the ER disrupter and impair hippocampal LTP, LTD, and spinogenesis. However, in physiological states, if we take into account the locally synthesized $\mathrm{E}_{2}$ in the hippocampus and the importance of fluctuations of local $E_{2}$ levels in cognitive circuits, a small amount of BPA could disturb the subtle regulation of $E_{2}$ level and then influence hippocampal LTP.

\section{Conclusions}

In summary, we demonstrated biphasic effects of BPA on LTP in DG region of rat hippocampus: exposure to BPA at a low dose $(100 \mathrm{nM})$ enhances LTP while to a high dose BPA $(1000 \mathrm{nM})$ inhibits LTP. The rapid facilitatory effect of low-dose BPA on hippocampal LTP requires membraneassociated ER and involves activation of ERK signaling pathway. Coadministration of $\mathrm{E}_{2}$ and $\mathrm{BPA}(100 \mathrm{nM})$ abolishes BPA-induced enhancement of LTP and $\mathrm{E}_{2}$-induced enhancement of baseline fEPSP, suggesting a complex interaction between BPA- and $\mathrm{E}_{2}$-mediated downstream pathways. Our investigation about hippocampal LTP implies that even nanomolar low doses of endocrine disrupters (e.g., BPA) could induce significant effects on hippocampal synaptic plasticity.

\section{Competing Interests}

All authors declare that they have no conflicts of interests.

\section{Authors' Contributions}

Xiaowei Chen and Yu Wang contributed equally to this study.

\section{Acknowledgments}

This work was supported by National Natural Science Foundation of China (U1503223, 81671089, 81472935, and 81172627), Zhejiang Provincial Natural Science Foundation of China (nos. LY15H090011, LY14H090004, and Z2090955), Ningbo Natural Science Foundation (2016A610086), and Ningbo municipal innovation team of life science and health (2015C110026) and sponsored by K. C. Wong Magna Fund in Ningbo University. 


\section{References}

[1] C. A. Richter, L. S. Birnbaum, F. Farabollini et al., "In vivo effects of bisphenol A in laboratory rodent studies," Reproductive Toxicology, vol. 24, no. 2, pp. 199-224, 2007.

[2] K. Kubo, O. Arai, M. Omura, R. Watanabe, R. Ogata, and S. Aou, "Low dose effects of bisphenol A on sexual differentiation of the brain and behavior in rats," Neuroscience Research, vol. 45, no. 3, pp. 345-356, 2003.

[3] C. R. Gonçalves, R. W. Cunha, D. M. Barros, and P. E. Martínez, "Effects of prenatal and postnatal exposure to a low dose of bisphenol A on behavior and memory in rats," Environmental Toxicology and Pharmacology, vol. 30, no. 2, pp. 195-201, 2010.

[4] X.-H. Xu, J. Zhang, Y.-M. Wang, Y.-P. Ye, and Q.-Q. Luo, "Perinatal exposure to bisphenol-A impairs learning-memory by concomitant down-regulation of N-methyl-d-aspartate receptors of hippocampus in male offspring mice," Hormones and Behavior, vol. 58, no. 2, pp. 326-333, 2010.

[5] S. Diaz Weinstein, J. J. Villafane, N. Juliano, and R. E. Bowman, "Adolescent exposure to Bisphenol-A increases anxiety and sucrose preference but impairs spatial memory in rats independent of sex," Brain Research, vol. 1529, pp. 56-65, 2013.

[6] D. Kumar and M. Kumar Thakur, "Perinatal exposure to bisphenol-A impairs spatial memory through upregulation of neurexin1 and neuroligin3 expression in male mouse brain," PLoS ONE, vol. 9, no. 10, Article ID e110482, 2014.

[7] R. N. Sadowski, L. M. Wise, P. Y. Park, S. L. Schantz, and J. M. Juraska, "Early exposure to bisphenol A alters neuron and glia number in the rat prefrontal cortex of adult males, but not females," Neuroscience, vol. 279, pp. 122-131, 2014.

[8] C. Wang, R. Niu, Y. Zhu et al., "Changes in memory and synaptic plasticity induced in male rats after maternal exposure to bisphenol A," Toxicology, vol. 322, pp. 51-60, 2014.

[9] Q. Zhang, X. Xu, T. Li et al., "Exposure to bisphenol-A affects fear memory and histone acetylation of the hippocampus in adult mice," Hormones and Behavior, vol. 65, no. 2, pp. 106-113, 2014.

[10] S. L. Neese, S. B. Bandara, and S. L. Schantz, "Working memory in bisphenol-A treated middle-aged ovariectomized rats," Neurotoxicology and Teratology, vol. 35, no. 1, pp. 46-53, 2013.

[11] R. Kuwahara, S. Kawaguchi, Y. Kohara, T. Jojima, and K. Yamashita, "Bisphenol A does not affect memory performance in adult male rats," Cellular and Molecular Neurobiology, vol. 34, no. 3, pp. 333-342, 2014.

[12] X. Xu, Y. Lu, G. Zhang et al., "Bisphenol A promotes dendritic morphogenesis of hippocampal neurons through estrogen receptor-mediated ERK1/2 signal pathway," Chemosphere, vol. 96, pp. 129-137, 2014.

[13] R. E. Bowman, V. Luine, H. Khandaker, J. J. Villafane, and M. Frankfurt, "Adolescent bisphenol-A exposure decreases dendritic spine density: role of sex and age," Synapse, vol. 68, no. 11, pp. 498-507, 2014.

[14] N. J. MacLusky, V. N. Luine, T. Hajszan, and C. Leranth, "The $17 \alpha$ and $17 \beta$ isomers of estradiol both induce rapid spine synapse formation in the Cal hippocampal subfield of ovariectomized female rats," Endocrinology, vol. 146, no. 1, pp. 287-293, 2005.

[15] T. Hajszan and C. Leranth, "Bisphenol A interferes with synaptic remodeling," Frontiers in Neuroendocrinology, vol. 31, no. 4, pp. 519-530, 2010.
[16] M. Yokosuka, R. Ohtani-Kaneko, K. Yamashita, D. Muraoka, Y. Kuroda, and C. Watanabe, "Estrogen and environmental estrogenic chemicals exert developmental effects on rat hypothalamic neurons and glias," Toxicology in Vitro, vol. 22, no. 1, pp. 1-9, 2008.

[17] T. Iwakura, M. Iwafuchi, D. Muraoka et al., "In vitro effects of bisphenol A on developing hypothalamic neurons," Toxicology, vol. 272, no. 1-3, pp. 52-58, 2010.

[18] N. Tanabe, T. Kimoto, and S. Kawato, "Rapid Ca(2+) signaling induced by Bisphenol A in cultured rat hippocampal neurons," Neuroendocrinology Letters, vol. 27, no. 1-2, pp. 97-104, 2006.

[19] N. Tanabe, H. Yoshino, T. Kimoto et al., "Nanomolar dose of bisphenol A rapidly modulates spinogenesis in adult hippocampal neurons," Molecular and Cellular Endocrinology, vol. 351, no. 2, pp. 317-325, 2012.

[20] M. Ogiue-Ikeda, N. Tanabe, H. Mukai et al., "Rapid modulation of synaptic plasticity by estrogens as well as endocrine disrupters in hippocampal neurons," Brain Research Reviews, vol. 57, no. 2, pp. 363-375, 2008.

[21] Y. Hasegawa, M. Ogiue-Ikeda, N. Tanabe et al., "Bisphenol a significantly modulates long-term depression in the hippocampus as observed by multi-electrode system," Neuroendocrinology Letters, vol. 34, no. 2, pp. 129-134, 2013.

[22] K. M. Frick, "Molecular mechanisms underlying the memoryenhancing effects of estradiol," Hormones and Behavior, vol. 74, pp. 4-18, 2015.

[23] X. Xu, T. Li, Q. Luo, X. Hong, L. Xie, and D. Tian, "Bisphenol-A rapidly enhanced passive avoidance memory and phosphorylation of NMDA receptor subunits in hippocampus of young rats," Toxicology and Applied Pharmacology, vol. 255, no. 2, pp. 221-228, 2011.

[24] I. Klyubin, Q. Wang, M. N. Reed et al., "Protection against A $\beta$ mediated rapid disruption of synaptic plasticity and memory by memantine," Neurobiology of Aging, vol. 32, no. 4, pp. 614-623, 2011.

[25] X. Chen, R. Lin, L. Chang et al., "Enhancement of long-term depression by soluble amyloid $\beta$ protein in rat hippocampus is mediated by metabotropic glutamate receptor and involves activation of p38MAPK, STEP and caspase-3," Neuroscience, vol. 253, pp. 435-443, 2013.

[26] M. R. Foy, J. Xu, X. Xie, R. D. Brinton, R. F. Thompson, and T. W. Berger, "17 $\beta$-estradiol enhances NMDA receptor-mediated EPSPs and long-term potentiation," Journal of Neurophysiology, vol. 81, no. 2, pp. 925-929, 1999.

[27] D. M. Vahaba and L. Remage-Healey, "Brain estrogen production and the encoding of recent experience," Current Opinion in Behavioral Sciences, vol. 6, pp. 148-153, 2015.

[28] S. M. Fernandez, M. C. Lewis, A. S. Pechenino et al., "Estradiolinduced enhancement of object memory consolidation involves hippocampal extracellular signal-regulated kinase activation and membrane-bound estrogen receptors," The Journal of Neuroscience, vol. 28, no. 35, pp. 8660-8667, 2008.

[29] A. M. Fortress, F. Lu, P. T. Orr, Z. Zhao, and K. M. Frick, "Estradiol-induced object recognition memory consolidation is dependent on activation of mTOR signaling in the dorsal hippocampus," Learning \& Memory, vol. 20, no. 3, pp. 147-155, 2013.

[30] X. Xu, Y. Ye, T. Li et al., "Bisphenol-A rapidly promotes dynamic changes in hippocampal dendritic morphology through estrogen receptor-mediated pathway by concomitant phosphorylation of NMDA receptor subunit NR2B," Toxicology and Applied Pharmacology, vol. 249, no. 2, pp. 188-196, 2010. 
[31] V. N. Luine, "Estradiol and cognitive function: past, present and future," Hormones and Behavior, vol. 66, no. 4, pp. 602-618, 2014.

[32] D. L. Korol and S. L. Pisani, "Estrogens and cognition: friends or foes?. An evaluation of the opposing effects of estrogens on learning and memory," Hormones and Behavior, vol. 74, pp. 105115, 2015.

[33] H. Mukai, T. Tsurugizawa, G. Murakami et al., "Rapid modulation of long-term depression and spinogenesis via synaptic estrogen receptors in hippocampal principal neurons," Journal of Neurochemistry, vol. 100, no. 4, pp. 950-967, 2007.

[34] L. C. Vedder, C. C. Smith, A. E. Flannigan, and L. L. Mcmahon, "Estradiol-induced increase in novel object recognition requires hippocampal NR2B-containing NMDA receptors," Hippocampus, vol. 23, no. 1, pp. 108-115, 2013.

[35] S. Kawato, "Endocrine disrupters as disrupters of brain function: a neurosteroid viewpoint," Environmental sciences : an international journal of environmental physiology and toxicology, vol. 11, no. 1, pp. 1-14, 2004.

[36] Y. Hasegawa, Y. Hojo, H. Kojima et al., "Estradiol rapidly modulates synaptic plasticity of hippocampal neurons: involvement of kinase networks," Brain Research, vol. 1621, pp. 147-161, 2015.

[37] N. J. MacLusky, T. Hajszan, and C. Leranth, "The environmental estrogen bisphenol A inhibits estradiol-induced hippocampal synaptogenesis," Environmental Health Perspectives, vol. 113, no. 6, pp. 675-679, 2005.

[38] A. Lacreuse, J. Chang, C. M. Metevier, M. Laclair, J. S. Meyer, and C. M. Ferris, "Oestradiol modulation of cognition in adult female marmosets (Callithrix jacchus)," Journal of Neuroendocrinology, vol. 26, no. 5, pp. 296-309, 2014.

[39] A. Chao, A. Paon, and L. Remage-Healey, "Dynamic variation in forebrain estradiol levels during song learning," Developmental Neurobiology, vol. 75, no. 3, pp. 271-286, 2015. 

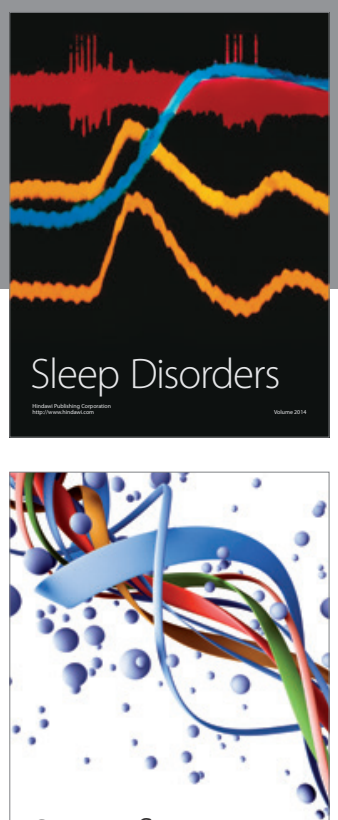

Scientifica
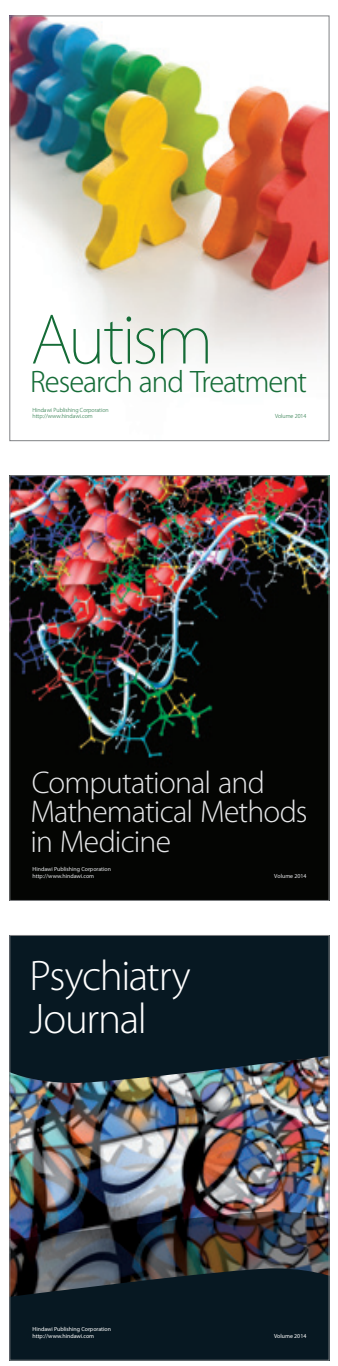
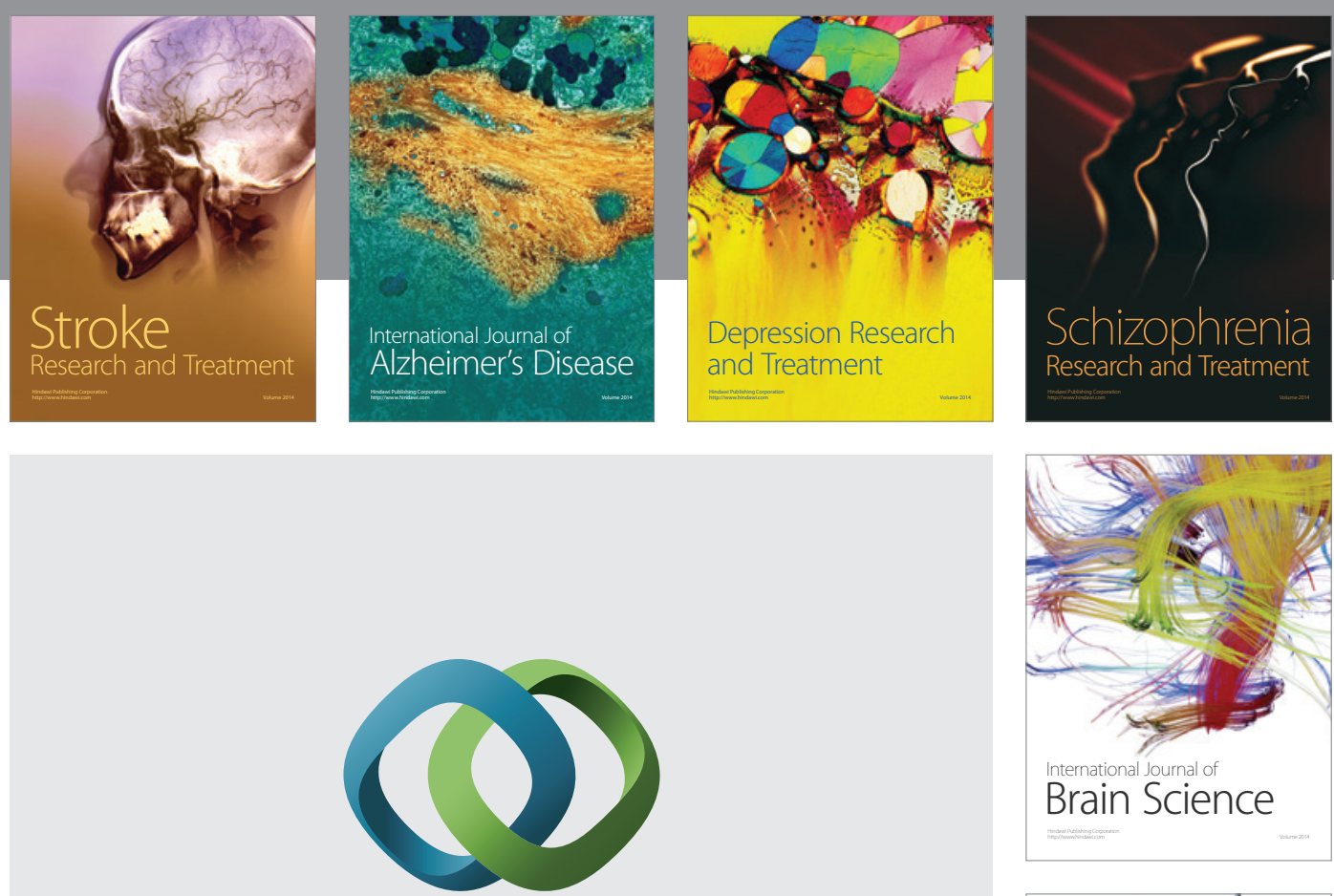

\section{Hindawi}

Submit your manuscripts at

https://www.hindawi.com
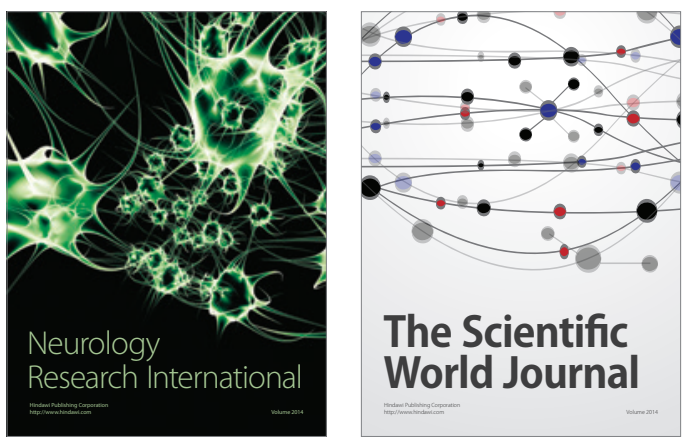

The Scientific World Journal

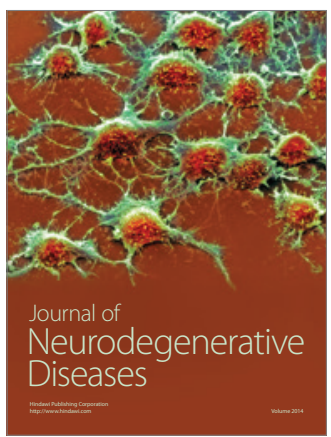

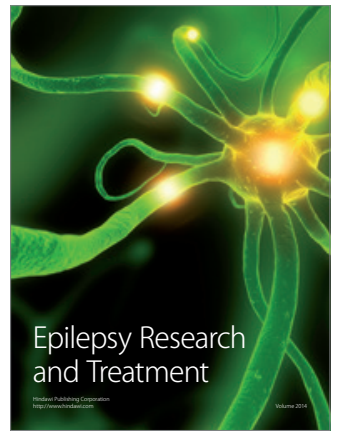

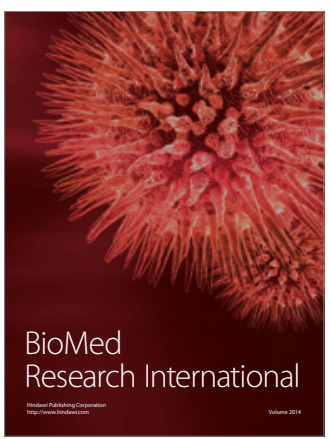

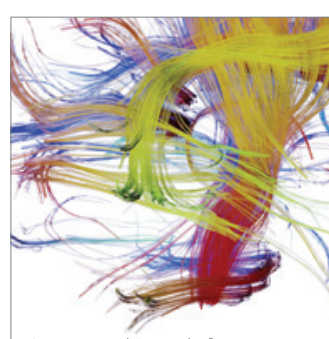

Brain Science

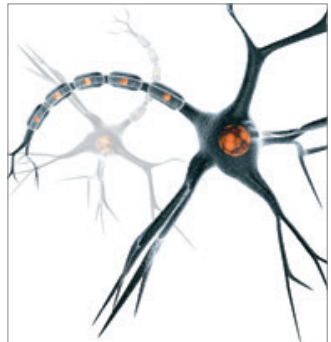

Neural Plasticity
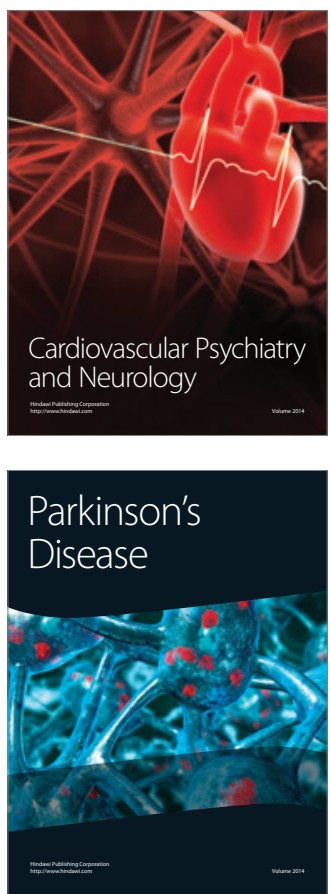